\title{
Mesenteric panniculitis
}

\author{
M. C. ORMiston \\ F.R.C.S. \\ H. THOMSON \\ M.S., F.R.C.S.
}

\author{
Gloucester Royal Hospital, Great Western Road, Gloucester
}

\begin{abstract}
Summary
A case of mesenteric panniculitis is presented, with a review of previous reports.
\end{abstract}

\section{Introduction}

Mesenteric panniculitis is a rare cause of pyrexia, abdominal pain, and an intra-abdominal mass.

\section{Case report}

A 31-year-old lorry driver was admitted with a 2-week history of fever, anorexia, and pain in the right loin. He looked ill, was pyrexial, and was tender in his right renal angle and right calf. There were no other physical signs. A provisional diagnosis of renal disease, possibly associated with a deep vein thrombosis, was made. However, an IVP and MSU were normal.

Investigations, including screens for occult carcinoma, auto-immune disease, infections, and bone marrow disorders, were all unremarkable except the ESR which was $124 \mathrm{~mm} / \mathrm{hr}$. One month after admission he developed a few subcutaneous nodules on his arms, biopsy of which showed non-specific fibrosis. Four weeks later the patient was still unwell and complained of increasingly severe back-ache. A diagnostic laparotomy was performed 3 months after presentation. A number of abnormalities were found. The folds of the mesentery were stuck together by flimsy vascular adhesions and fibrin. Similar adhesions were present between the liver and diaphragm. The base of the ileal mesentery was greatly thickened and contained enlarged rubbery nodules which, on section, revealed a variegated yellow appearance. Lymph nodes, liver and peritoneal specimens were taken for histology and culture. Histology showed active fibrosis of the peritoneum but no specific features. The lymphoid histiocytes contained finely divided fat, an appearance consistent with lymph nodes draining an area of active fat inflammation and necrosis. The liver biopsy showed a non-specific increase of inflammatory cells. Culture for organisms including tuberculosis was negative.

\section{Discussion}

Mesenteric panniculitis is a rare condition usually affecting the middle-aged. Presenting features may include abdominal or back pain, a mass, vomiting, bowel disturbance, passage of blood per rectum and pyrexia (Ogden, Bradburn and Rives, 1965; Durst et al., 1977).

Investigations are often normal and are of no diagnostic help.

Pathological findings range from lumpy thickening of the ileal mesentery, which has yellowish discoloured patches and microscopic infiltration of the fat by lymphocytes, macrophages, and foreign body giant cells, to a retracted, scarred, and adherent mesentery with fat necrosis, fibrosis, and calcification (Ogden et al., 1965). The disease is generally confined to the small bowel mesentery, but can involve retroperitoneal structures and the sigmoid mesocolon (Durst et al., 1977).

This condition has been described as retractile mesenteritis, which represents an advanced stage of the disease with scarring and fibrosis (Soergel and Hensley, 1966), and also mesenteric manifestations of Weber Christian disease (Herrington, Edwards and Grossman, 1961), the characteristic subcutaneous nodules of inflamed adipose tissue found in Weber Christian disease being absent. Foci of fat inflammation in the retroperitoneal, pericardial and mediastinal tissues in Weber Christian disease are occasionally reported post mortem (Steinberg, 1953; Milner and Mitchinson, 1965). However, the course of mesenteric panniculitis is usually benign (Ogden et al., 1965; Durst et al., 1977).

The aetiology of mesenteric panniculitis is unknown. A wide variety of other conditions have occasionally been found in association.

Surgical intervention is generally confined to laparotomy and biopsy. By-pass or resection of obstructed bowel may be required. Steroids are said to afford symptomatic relief in some instances.

The presentation of this patient was not atypical, except that his ESR was remarkably high. However, he did not respond to prednisone and remains ill and in pain 15 months after laparotomy. The subcutaneous nodules are of interest in view of the 
comparisons drawn between mesenteric panniculitis and Weber Christian disease. Whether or not the 2 conditions are expressions of the same underlying pathological process is uncertain. The outlook for patients with fat inflammation apparently confined to the subcutaneous tissue or ileal mesentery is good, but there is evidence that more widespread disease is associated with a considerable increase in morbidity and mortality.

\section{Acknowledgments}

The authors wish to thank Anne Stinchcombe for typing the manuscript.

\section{References}

Durst, A.L., Freund, H., Rosenmann, E. \& Birnbaum, D. § (1977) Mesenteric panniculitis: Review of literature and $C$ presentation of cases. Surgery, 81, 203.

Herrington, J.L., Edwards, W.H. \& Grossman, L.A. (1961) Mesenteric manifestations of Weber Christian disease. Annals of Surgery, 154, 949.

Milner, R.D.G. \& Mitchinson, M.J. (1965) Systemic Weber Christian disease. Journal of Clinical Pathology, 118, 150.

Ogden, W.W., BradburN, D.M. \& Rives, J.D. (1965) Mesenteric panniculitis. Annals of Surgery, 161864

Soergel, K.H. \& Hensley, G.T. (1966) Fatal mesenteric panniculitis. Gastroenterology, 51, 529.

STEINBERG, B. (1953) Systemic nodular panniculitis. American Journal of Pathology, 29, 1059. 